\title{
Search for endophytic diazotrophs in barley seeds
}

\author{
Myriam S. Zawoznik ${ }^{1}$, Susana C. Vázquez ${ }^{2}$, \\ Silvana M. Díaz Herrera ${ }^{1}$, María D. Groppa ${ }^{1}$ \\ ${ }^{1}$ Cátedra de Química Biológica Vegetal, Departamento de Química Biológica, \\ Universidad de Buenos Aires, Buenos Aires, Argentina. \\ ${ }^{2}$ Cátedra de Microbiología Industrial y Biotecnología, Departamento de Microbiología, Inmunología y \\ Biotecnología, Facultad de Farmacia y Bioquímica, Universidad de Buenos Aires, \\ Buenos Aires, Argentina.
}

Submitted: February 18, 2013; Approved: September 9, 2013.

\begin{abstract}
Eight endophytic isolates assigned to Pseudomonas, Azospirillum, and Bacillus genera according to pheno-genotypic features were retrieved from barley seeds under selective pressure for nitrogen-fixers. Genetic relationships among related isolates were investigated through RAPD. Six isolates displayed nitrogen-fixing ability, while all could biosynthesize indolacetic acid in vitro and showed no antibiosis effects against Azospirillum brasilense Az39, a recognized PGPR.
\end{abstract}

Key words: Azospirillum, Bacillus, Pseudomonas, PGPR, RAPD.

Inoculation of crops using biofertilizers containing different plant growth promoting rhizobacteria (PGPR) has gained great acceptance over last years. This technology not only may result in increased crop yields, but it is also expected to reduce the need for chemical fertilizers and pesticides, allowing a more sustainable scheme of agricultural practice (Kumar et al., 2011; Rai, 2006).

Malting barley cultivated area in Buenos Aires province, Argentina, has been growing during last decades. An increasing international demand, good yields and a shorter life cycle (which allows the anticipation of soybean sowing), as compared to other traditional winter crops, have been identified as the main factors leading to this trend (Bragachini and Peiretti, 2012). Since water and nitrogen availability are usually determinant factors for barley yield in this region, the use of Azospirillum-based inoculants arises as a reasonable biotechnological approach to cope, at least in part, with these constraints, as they are expected to promote root system development and, consequently, to allow better access to soil water and dissolved nutrients. On another hand, these products may contribute to $\mathrm{N}$ plant nutrition through biological fixation.

In previous experiments we inoculated barley seedlings with the collection strain A. brasilense Az39 and ob- served that Azospirillum counts in the roots of inoculated plants (based on the standard MPN procedure on semisolid malate enrichment medium) were generally below those reported for other plant species studied in Azospirillum inoculation trials. At the same time, development of bacterial communities in the aforementioned culture medium for control units (uninoculated roots) was frequently corroborated, despite the fact that all seeds had been surfacedisinfected before sowing (unpublished data). These observations led us to suspect that barley seeds could contain endophytic diazotrophic microorganisms that eventually might compete with introduced PGPR during colonization of the rhizospheric niche. In this sense, it was reported that rice seed endophytes inhibited early colonization of rice roots by $A$. brasilense (Bacilio-Jiménez et al., 2001).

In this work, our goal was to detect and to identifiy endophytic diazotrophs in the seeds of three barley cultivars recommended for malting barley production in Buenos Aires province (Argentina) and to test under laboratory conditions their nitrogenase activity and indolacetic acid (IAA) production, to gain a basic approach of their plant growth promoting potential. Additionally, we examined possible antagonisms between these endophytic microorganisms and Azospirillum brasilense strain Az39, a well-

Send correspondence to M.D. Groppa. Cátedra de Química Biológica Vegetal, Facultad de Farmacia y Bioquímica, Universidad de Buenos Aires, Junín 956, (1113) Ciudad Autónoma de Buenos Aires, Argentina. E-mail: mgroppa@ffyb.uba.ar. 
known strain from a culture collection which is included in the formulation of several biofertilizers commercialized in Argentina.

Seeds of the malting barley cultivars Scarlett, Quilmes Carisma, and Josefina INTA were surface disinfected (70\% ethanol $5 \mathrm{~min}$, followed by $5 \%$ sodium hypochlorite containing three drops of Tween 20 for $15 \mathrm{~min}$ ) and thoroughly rinsed with sterilized distilled water. Then, seeds were longitudinally split and crushed in the upper part of a $5-\mathrm{mL}$ vial containing $\mathrm{NFb}$ semisolid medium (Dobereiner, 1980), and incubated at $30{ }^{\circ} \mathrm{C}$ for 3 days. Disinfected uncrushed seeds were also placed into $\mathrm{NFb}$ vials and incubated in the same conditions. NFb vials showing turbidity and/or pellicle and $\mathrm{pH}$ rise were subcultured on Petri dishes containing RC medium (Rodríguez-Cáceres, 1982). Reddish colonies displaying different phenotypes were selected and transferred to $\mathrm{NFb}$ vials. After 3 days of incubation, vials showing turbidity and/or a whitish pellicle or a faint veil, and $\mathrm{pH}$ rise and a dominant population of motile rods observed under optic microscopy were grown on RC plates and then in $\mathrm{NFb}$ semisolid medium repeated times until pure cultures on RC plates were obtained.

Gram staining, cell shape, colony morphology and routine biochemical tests guided preliminary identification of the isolates obtained (Holt et al., 1994; University of Idaho webpage, 2011) and 16S rRNA gene sequencing was carried out to complete identification at genus level. Total DNA was extracted from randomly chosen colonies of each selected isolate using the Illustra ${ }^{\circledR}$ Blood Genomic Prep Mini Spin Kit (GE Healthcare). A fragment of around $1500 \mathrm{bp}$ of the 16S rRNA gene was amplified using the universal primers $27 \mathrm{~F}$ and $1492 \mathrm{R}$. PCR products were purified with the Wizard ${ }^{\mathbb{B}}$ PCR purification kit (Promega) and sequenced at the Genomic Unit of the Biotechnology Institute of CNIA-INTA using a capillary automatic sequencer model ABI3130XL (Applied Biosystems, USA). The Naïve Bayesian Classifier utility (Wang et al., 2007) from the RDP Release 10 (http://rdp.cme.msu.edu) was used to assign the obtained sequences into the new bacterial taxonomy at genus level with $95 \%$ of confidence. The Seqmatch utility (type strains) from the same RDP website and the Eztaxon-e utility from the EzBioCloud platform (http://eztaxon-e.ezbiocloud.net) were used to find closest sequences according to their 16S rRNA gene sequence similarity, in order to suggest most probable affiliations of the isolates obtained at species level.

A RAPD analysis using three different primers was used to study the genetic variability between isolates linked to the same or closely related species. Four independent PCR reactions were carried out using random primers OPA3 (5'-AGTCAGCCAC-3'), OPA9 (5'-GGGTAA CGCC-3') and OPA18 (5'-AGGTGACCGT-3') (Operon Techn. Inc. Alameda, CA) alone and a combination of the three primers. Isolates would be categorized as unrelated strains in the presence of RAPD profiles differing by three or more bands. Differences in band intensity (at a given position) were not taken into account for the differentiation of isolates.

IAA biosynthetic ability of the isolates obtained was estimated by Salkowski colorimetric technique using OAB medium (Okon et al., 1977) amended with $100 \mu \mathrm{g} \mathrm{mL} \mathrm{m}^{-1}$ of L-tryptophan (Glickmann and Dessaux, 1995). Nitrogen fixing ability of these strains was determined by the acetylene reduction assay (Hardy et al., 1968).

The potential antagonistic effects of the isolates obtained against Azospirillum brasilense strain Az39 were investigated using an adaptation of the double agar layer plate assay described by Muñoz-Rojas et al. (2005). All determinations were performed from three independent experiments and analytical measurements were done with a minimum of three replicates.

Between two and six scarlet or reddish colonies with different appearance could be recognized on each RC plate, while no microbial development was observed in the $\mathrm{NFb}$ vials where disinfected uncrushed seeds were deposited. Further purification of these colonies through consecutive passages between semisolid NFb and solid RC media finally rendered a total of eight isolates: three of them came from cultivar Scarlett (isolates 21, 22, and 23), two from cultivar Quilmes Carisma (isolates 24 and Q), and three from cultivar Josefina INTA (isolates 2, 3, and 10).

The isolates obtained were affiliated to three bacterial genera, Bacillus, Pseudomonas and Azospirillum (Table 1). These genera are traditionally regarded as root-associated or soil-dwelling bacteria rather than true endophytic diazotrophs (James and Olivares, 1997; Reinhardt et al., 2008). Endophytic colonization and plant growth promoting traits for different members of Bacillus and Pseudomonas were largely documented (Botelho Praça et al., 2012; Compant et al., 2011; Mishra et al., 2009; Zhao et al., 2011). Using microscopy coupled with immunogold labeling, important diazotrophs like Herbaspirillum seropedicae and Azospirillum brasilense were found within cavities in surface-sterilized rice seeds by Baldani et al. (1993). These authors suggested that microorganisms may be transferred from generation to generation via seeds. Sundaram et al. (1988) also reported Azospirillum spp. in the seeds of turfgrass. As far as we know, the finding of Azospirillum as a barley seed endophyte has not been reported before.

The partial sequences of the 16S rRNA gene of isolates 10 and 24 shared $100 \%$ identity and were closely related to the sequence of the type strain of $A$. doebereinerae ( $99 \%$ identity), suggesting that these isolates probably belong to this species. In a similar way, we found that isolate 21 is related with high probability to Bacillus pumilus and isolate 23 to Bacillus thuringiensis, according to the $16 \mathrm{~S}$ rRNA gene sequence analysis. Regarding Pseudomonas isolates, given the high percentage of identity between the 16S rRNA gene sequences of the different type strains 


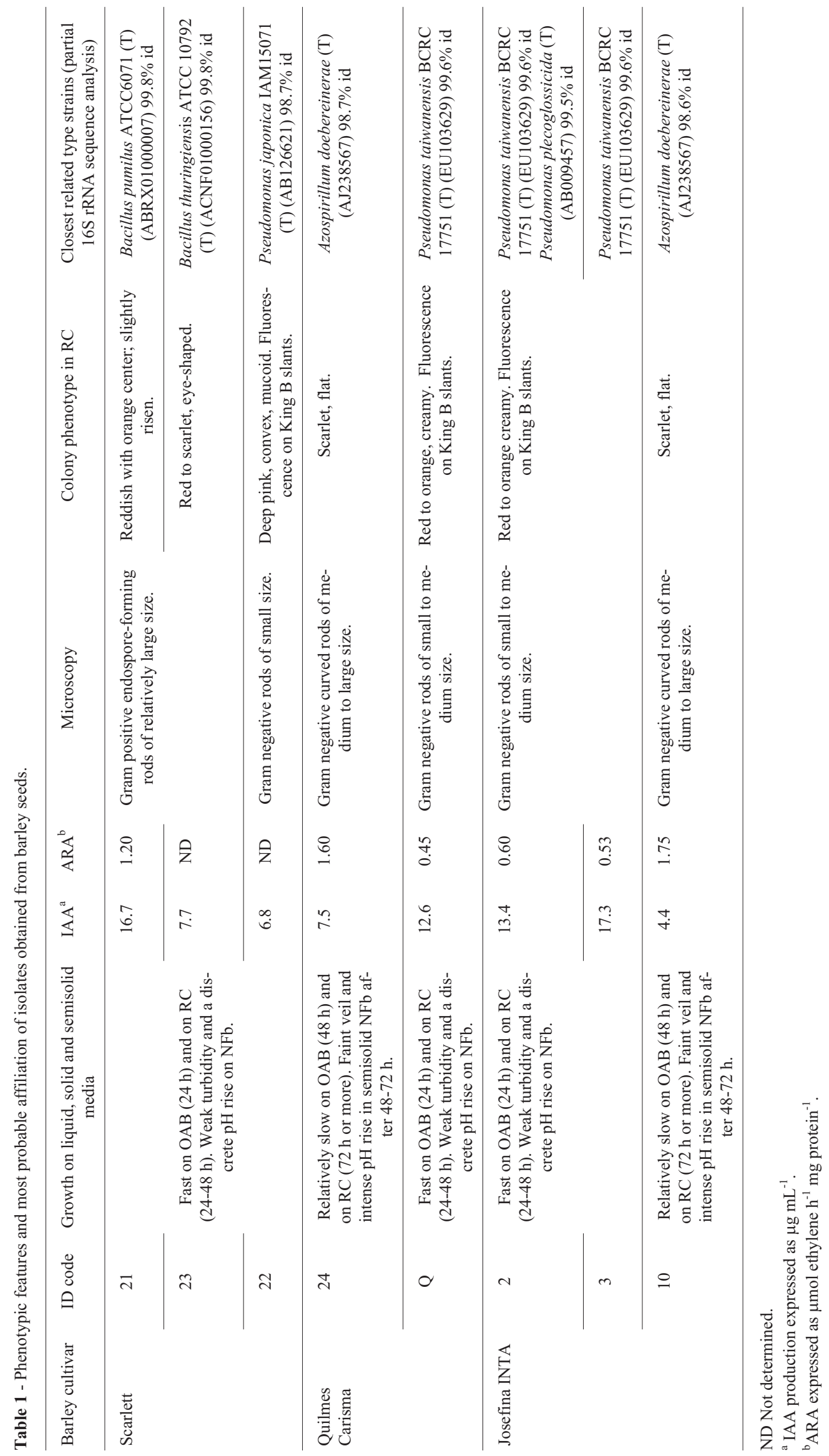


within the genera, we can only state that isolates $\mathrm{Q}, 2$, and 3 are related to the $P$. putida group according to the phylogenetic analysis reported by Mulet et al. (2010), while isolate 22 is related to the $P$. lutea group.

The RAPD analysis with primers OPA 3, OPA 9, and OPA 18, used independently or combined in the same PCR reaction, did not differentiate between isolates ascribed to the same Pseudomonas group (Figure 1, lanes 2-13), suggesting that they could correspond to the same strain, even when they were obtained from different cultivars (Quilmes Carisma and Josefina INTA). The same was observed for Azospirillum isolates (Figure 1, lanes 14-21). In other words, our results did not reveal genotypic variation among isolates related to the same bacterial species or phylogenetic group, but suggest an interesting relation between the taxonomic affiliation of the microorganisms isolated and the cultivar where they came from, as isolates retrieved from the seeds of Scarlett (a traditional European spring and winter barley variety) did not show overlap with the isolates obtained from the seeds of the Argentinian improved cultivars Quilmes Carisma (lodging resistant) and Josefina INTA (short cycle, cold resistant), where we corroborated the presence of the same or quite similar genotypes on comparing isolates recovered through the culturedependent protocol here followed.

All the isolates obtained were able to produce IAA in OAB medium amended with tryptophan. Isolates that affiliated in the $P$. putida group and the isolate with maximum identity with $B$. pumilus displayed the highest in vitro IAA production levels, while one of the isolates with maximum identity with $A$. doebereinerae type strain (isolate 10) showed the lowest. The ability to produce IAA using tryptophan as precursor is a well-known feature of many members of the bacterial genera here identified (Spaepen et al., 2007). Regarding this biosynthetic ability in our isolates, a broader spectrum of culture conditions should be investigated before drawing definite conclusions.

Two isolates failed to grow in NFb once completely purified and were therefore considered non-nitrogen fixers. The other isolates exhibited different ARA levels (Table 1). These results suggest the existence of cometabolic interactions among seed endophytic communities, which may have allowed the survival of non-fixing organisms in the absence of a nitrogen source at the initial steps of our isolation protocol. The ability to fix nitrogen, which has long been considered to be absent from the Pseudomonas genus, is now well recognized in several strains of Pseudomonas stutzeri (Desnoues et al., 2003; Yan et al., 2008) and in Pseudomonas azotifigens sp. nov. (Hatayama et al., 2005), both included in the P.stutzeri group, P. aeruginosa lineage, according to Mulet et al. (2010). Our Pseudomonas isolates, with weak or null ARA, philogenetically diverged from the aforementioned microorganisms, as they were linked to the $P$. fluorescens lineage.

To sum up, through this research we proved that barley seeds host bacterial inhabitants that eventually may contribute to early plant growth through different plant growth promoting abilities, like indolacetic acid production and/or nitrogen fixation. On the other hand, we observed that these microorganisms had no antibiosis effects against A.brasilense Az39. Therefore, the low Azospirillum counts verified in previous experiments where barley seedlings were inoculated with this strain should not be imputed to active antibiosis mechanisms of these endophytes. The possible activity of other endophytes excluded under our isolation protocol, and eventual differences in the competitive ability of the endophytic communities under natural conditions as compared to introduced microorganisms, may rather account for our previous findings. Rizosphere colonization is an intrincate process that certainly deserves further investigation efforts.

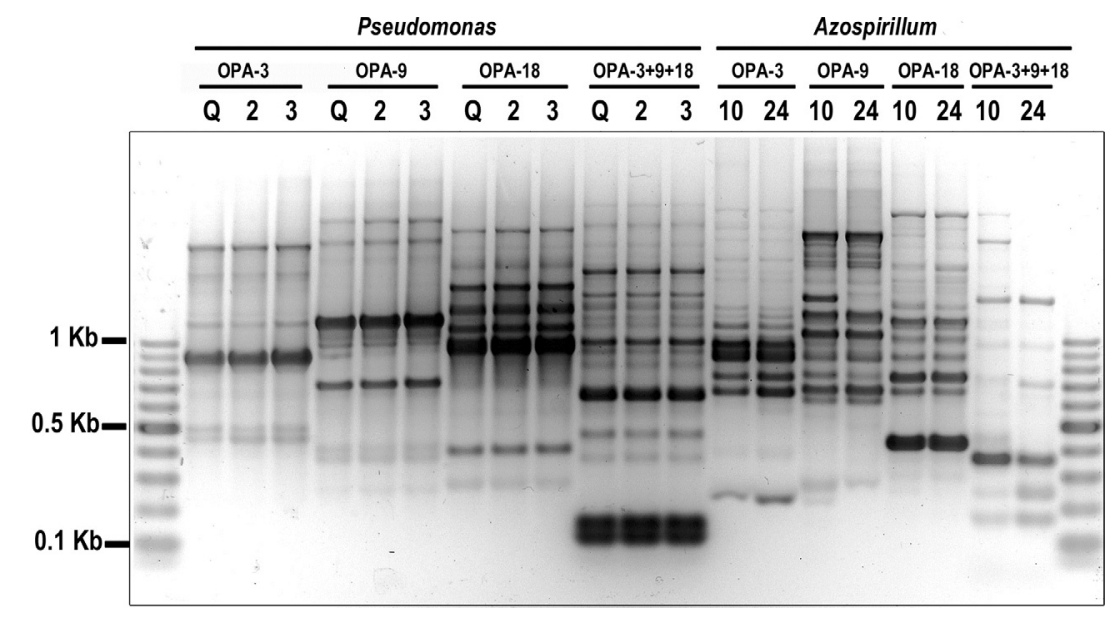

Figure 1 - RAPD analysis of Azospirillum and Pseudomonas (P. putida group) isolates. RAPD amplified products separated on $2.5 \%$ agarose gel and stained with GelRed ${ }^{\circledR}$. A 100-1000 bp DNA ladder was used as molecular weight standard (Lanes 1 and 22). DNA was amplified with random primers OPA 3, OPA 9 and OPA 18, used independently or combined in the same PCR reaction, as described in the text. 


\section{Acknowledgments}

This work was supported by the University of Buenos Aires (UBACYT 20020090200159) and CONICET (PIP 0097). María D. Groppa and Susana Vázquez are researchers at the Consejo Nacional de Investigaciones Científicas y Técnicas (CONICET). We thank Ing. Agr. Alejandro Perticari (IMYZA-INTA) and Ing. Agr. Fernando Giménez (INTA-Bordenave Experimental Station) for providing the Azospirillum brasilense Az39 strain and the seeds used in this work.

\section{References}

Bacilio-Jiménez M, Aguilar-Flores S, del Valle MV, Pérez A, Zepeda A, Zenteno E (2001) Endophytic bacteria in rice seeds inhibit early colonization of roots by Azospirillum brasilense. Soil Biol Biochem 33:167-172.

Baldani VLD, James EK, Baldani JI, Döbereiner J (1993) Colonization of rice by the nitrogen-fixing bacteria Herbaspirillum spp and Azospirillum brasilense. In: Palacios, R., Mora, J., Newton, W.E. (eds). New Horizons in Nitrogen Fixation. Kluwer Academic, Dordrecht, pp 705.

Botelho Praça L, Menezes Mendes Gomes AC, Cabral G, Soares Martins É, Ryoiti Sujii E, Gomes Monnerat R (2012) Endophytic colonization by Brazilian strains of Bacillus thuringiensis on cabbage seedlings grown in vitro. BTR 3:11-19.

Bragachini M, Peiretti J (2012) Cebada cervecera, el 2. ${ }^{\text {do }}$ cultivo de invierno en Argentina con franca expansión. Comencemos sin pérdidas el camino hacia la industria. Precop INTA.

http://www.cosechaypostcosecha.org/data/articulos/cosech a/CebadaCerveceraFrancaExpansion.asp.

Compant S, Mitter B, Colli-Mull JG, Gangl H, Sessitsch A (2011) Flowers, berries, and seeds: Identification of cultivable bacteria, comparison with other plant parts, and visualization of niches of colonization. Microb Ecol 62:188-197.

Desnoues N, Lin M, Guo X, Ma L, Carreño-Lopez R, Elmerich C (2003) Nitrogen fixation genetics and regulation in a Pseudomonas stutzeri strain associated with rice. Microbiology 149:2251-2262.

Dobereiner J (1980) Forage grasses and grain crops. In: Bergersen, F.J. (ed) Methods for evaluating biological nitrogen fixation. John Wiley \& Sons Ltd., New York, pp 535-555.

Glickmann E, Dessaux Y (1995) A critical examination of the specificity of the Salkowski reagent for indolic compounds produced by phytopathogenic bacteria. Appl Environ Microbiol 61:793-796.

Hardy RWF, Holsten RD, Jackson EK, Burns RC (1968) The acetylene-ethylene assay for $\mathrm{N}_{2}$ fixation: laboratory and field evaluation. Plant Physiol 43:1185-1207.

Hatayama K, Kawai S, Shoun H, Ueda Y, Nakamura A (2005) Pseudomonas azotifigens sp. nov., a novel nitrogen-fixing bacterium isolated from a compost pile. Int J Syst Evol Micr 55:1539-1544.

Holt JG, Krieg NR, Sneath PHA, Staley JT, Williams ST (1994) Bergey's Manual of Determinative Bacteriology. $9^{\text {th }}$ edition. Williams \& Wilkins, Baltimore.
James EK, Olivares FL (1997) Infection and colonization of sugar cane and other graminaceous plants by endophytic diazotrophs. Crit Rev Plant Sci 17:77-119.

Kumar A, Prakash A, Johri BN (2011) Bacillus as PGPR in crop ecosystem. In: Maheshwari, D.K. (ed). Bacteria in Agrobiology: Plant Nutrient Management. Springer-Verlag, Berlin, pp 37-59.

Mishra PK, Mishra S, Selvakumar G, Bisht JK, Kundu S, Gupta HS (2009) Coinoculation of Bacillus thuringeinsis-KR1 with Rhizobium leguminosarum enhances plant growth and nodulation of pea (Pisum sativum L.) and lentil (Lens culinaris L.). World J Microbiol Biotechnol 25:753-761.

Mulet M, Lalucat J, García-Valdés E (2010) DNA sequencebased analysis of the Pseudomonas species. Environ Microbiol 12:1513-1530.

Muñoz-Rojas J, Fuentes-Ramírez LE, Caballero-Mellado J (2005) Antagonism among Gluconacetobacter diazotrophicus strains in culture media and in endophytic association. FEMS Microbiol Ecol 54:57-66.

Okon Y, Albrecht SL, Burris RH (1977) Methods for growing Spirillum lipoferum and for counting it in pure culture and in association with plants. Appl Environ Microbiol 33:85-88.

Rai MK (2006) Handbook of Microbial Biofertilizers. The Haworth Press, New York.

Reinhardt E, Ramos PL, Manfio GP, Barbosa HR, Pavan C, Moreira-Filho CA (2008) Molecular characterization of nitrogen-fixing bacteria isolated from Brazilian agricultural plants at São Paulo state. Braz J Microbiol 39:414-422.

Rodríguez-Cáceres EA (1982) Improved medium for isolation of Azospirillum spp. Appl Environ Microbiol 44:990-991.

Spaepen S, Vanderleyden J, Remans R (2007) Indole-3-acetic acid in microbial and microorganism-plant signaling. FEMS Microbiol Rev 31:1-24.

Sundaram S, Arunakumari A, Klucas RV (1988) Characterization of Azospirilla isolated from seeds and roots of turf grass. Can J Microbiol 34: 212-217.

University of Idaho (2011) Differentiation via Gram stains and cell morphology. Gram stain \& morphological flowchart. Available at: http://www.uiweb.uidaho.edu/micro_biology/250/IDFlowcharts.pdf. Accessed 30 May 2012.

Wang Q, Garrity GM, Tiedje JM, Cole JR (2007) Naïve Bayesian classifier for rapid assignment of rRNA sequences into the new bacterial taxonomy. Appl Environ Microbiol 73:52615267.

Yan Y, Yang J, Dou Y, Chen M, Ping S, Peng J, Lu W, Zhang W, Yao Z, Li H, Liu W, He S, Geng L, Zhang X, Yang F, Yu H, Zhan Y, Li D, Lin Z, Wang Y, Elmerich C, Lin M, Jin Q (2008) Nitrogen fixation island and rhizosphere competence traits in the genome of root-associated Pseudomonas stutzeri A1501. Proc Natl Acad Sci USA 105:7564-7569.

Zhao L, Xu Y, Sun R, Deng Z, Yang W, Wei G (2011) Identification and characterization of the endophytic plant growth prompter Bacillus cereus strain MQ23 isolated from Sophora alopecuroides root nodules. Braz J Microbiol 42:567-575.

All the content of the journal, except where otherwise noted, is licensed under a Creative Commons License CC BY-NC. 